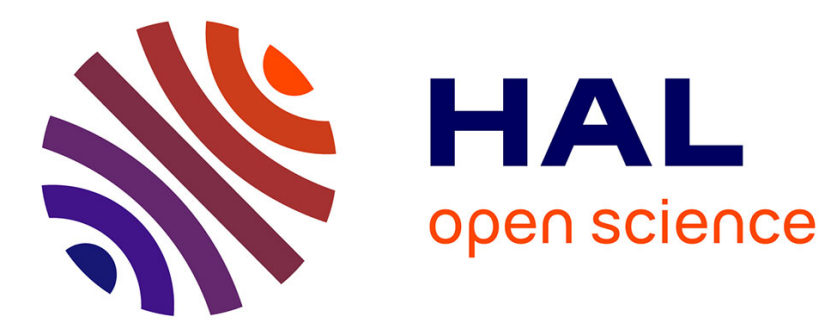

\title{
DEPOTS CHIMIQUES EN PHASE VAPEUR DE CARBURE DE BORE SUR CARBURE DE TUNGSTENE FRITTE ET SUR ACIER
}

S. Lartigue, D. Cazajous, G. Male

\section{- To cite this version:}

S. Lartigue, D. Cazajous, G. Male. DEPOTS CHIMIQUES EN PHASE VAPEUR DE CARBURE DE BORE SUR CARBURE DE TUNGSTENE FRITTE ET SUR ACIER. Journal de Physique Colloques, 1986, 47 (C1), pp.C1-197-C1-202. 10.1051/jphyscol:1986130 · jpa-00225559

\section{HAL Id: jpa-00225559 https://hal.science/jpa-00225559}

Submitted on 1 Jan 1986

HAL is a multi-disciplinary open access archive for the deposit and dissemination of scientific research documents, whether they are published or not. The documents may come from teaching and research institutions in France or abroad, or from public or private research centers.
L'archive ouverte pluridisciplinaire HAL, est destinée au dépôt et à la diffusion de documents scientifiques de niveau recherche, publiés ou non, émanant des établissements d'enseignement et de recherche français ou étrangers, des laboratoires publics ou privés. 
JOURNAL DE PHYSIQUE

Colloque $C 1$, supplément au $n^{\circ} 2$, Tome 47 , février 1986

page $\mathrm{C} 1-197$

DEPOTS CHIMIQUES EN PHASE VAPEUR DE CARBURE DE BORE SUR CARBURE DE
TUNGSTENE FRITTE ET SUR ACIER

\author{
S. LARTIGUE, D. CAZAJOUS ${ }^{*}$ et G. MALE* * \\ E.T.C.A., Avenue Prieur de la Côte d'Or, F-94114 Arcueil, France \\ * Sté Diamant Boart, Div. Pétrole, z.I. Montardon, \\ F-64121 Serres-Castet, France \\ ${ }^{*}$ *C.N.R.S., Laboratoire des Ultra-Réfractaires, B.P. 5 , \\ F-66120 odeillo, France
}

\begin{abstract}
Résumé - La synthèse des carbures de bore par vapodéposition chimique à partir de mélanges de $\mathrm{BCl}_{3}, \mathrm{CH}_{4}$ et $\mathrm{H}_{2}$ est appliquée au revêtement de carbures de tungstène frittés èt aux aciers. Les interactions avec ces substrats et notamment la diffusion du bore avec formation d'une zone perturbée sont examinés. L'adhérence, Ia microdureté et des tests de forage permettent d'envisager des applications dans les domaines de l'usinage et de la résistance à l'usure.
\end{abstract}

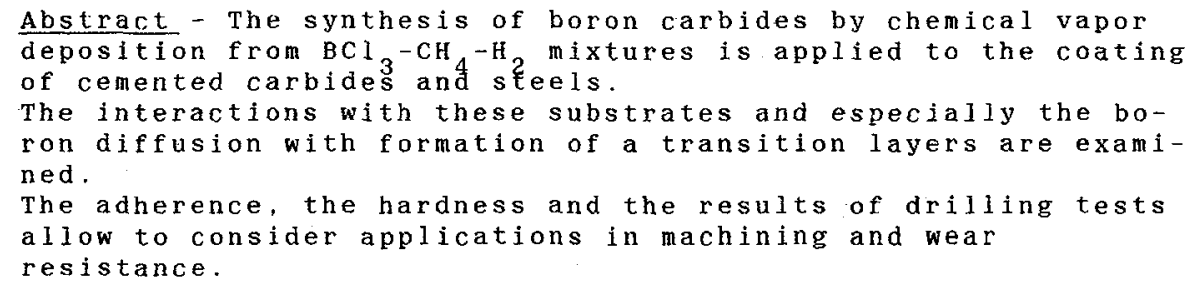

\title{
I - INTRODUCTION
}

Le carbure de bore est un matériau bien connu sous la forme de produits frittés pour ses propriétés exceptionnelles qui sont à la base de ses applications: pièces résistant à l'usure, abrasifs, barres de contrôle des réacteurs nuclèaires, blindages, etc... Une synthèse des travaux bibliographiques a récemment été faite sur le sujet par Thevenot et col./1/,/2/.

Le présent travail constitue une tentative pour améliorer la dureté et la résistance à l'usure superficielles des carbures frittés et des aciers.

\section{I - CONDITIONS DE REVETEMENT.}

Les revetements de carbure de bore sont préparés dans un réacteur à parois froides par dépôt chimique à partir de mêlanges gazeux de $\mathrm{BCl}_{3}, \mathrm{CH}_{4}$ et $\mathrm{H}_{2}$ sous une pression totale de $10 \mathrm{mbar}$. Les substrats d'étude sônt dešs cylindres de $W C+$ co ou d'acier de diamètres respectifs 13 et $16 \mathrm{~mm}$ et de $5 \mathrm{~mm}$ d'épaisseur.

Pour éviter une transformation des substrats (sublimation du cobalt, fusion de l'eutectique $\mathrm{Fe}-\mathrm{Fe}{ }_{2} \mathrm{~B}$, etc...) les dépôts sont effectués à basse température $(1300 \mathrm{k})^{2}$. 
Dans ces conditions, si on fait croitre par exemple la teneur relative en $\mathrm{CH}_{4}$ du mélange gazeux, une étude antérieure/3/ a montré que I'on obtenait successivement une phase quadratique $\left(B_{50} C_{2}\right)$, un diphasé ( $\left.\mathrm{B}_{50} \mathrm{C}_{2}+\mathrm{B}_{13} \mathrm{C}_{2} "\right)$ la solution solide "B ${ }_{13} \mathrm{C}_{2}$ et ứn deuxième

diphasê ( "B ${ }_{13} \mathrm{C}_{2}$ " + $\left.\mathrm{X}\right)$.

De façon. concomittante, la teneur en carbone varie de 2 à $18 \%$ at., et la microdureté knoop (sous une charge de $100 \mathrm{~g}$ ) de 23 à 49 GPa.

\section{II - INTERACTIONS AVEC LE SUBSTRAT.}

Au cours de l'opération de dépôt, a lieu une importante diffusion du bore à la surface du substrat. Des profils élémentaires obtenus par spectrométrie d'émission à décharge luminescente (S.D.L.) mettent en évidence ce phénomène (fig.1 et 2). Le bore réagit avec son environnement et forme des composés qui jouent un rôle prépondérant dans l'adhérence du dépôt. Des migrations d'autres éléments sont également observées.

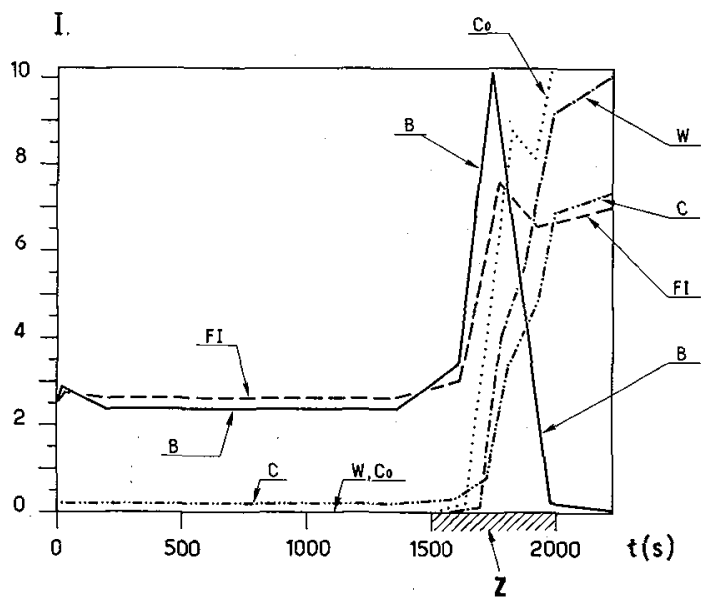

I.

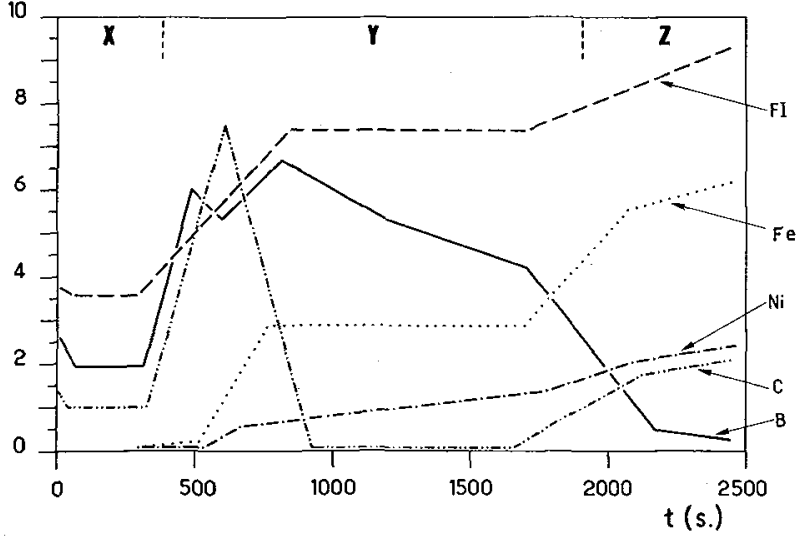

Fig:1 - Evolution des concentrations élémentaires suivant la normale à la surface (S.D.L.). Dépôt de carbure de bore sur WC $+30 \% \mathrm{Co}$. I : en unités arbitraires, $E I=f a i s c e a u$ intégral, $z=$ zone de diffusion.

Fig.1 - Evolution of the in-depth elementary concentration (G.D.O. S.). Boron carbide deposit on WC $+30 \%$ Co. I : arbitrary units, F.I. = integral beam, $Z=$ diffusion Iayer.

Fig. 2 - Profils de concentrations (S.D.L.). Dépôt sur acier 35 NCD 16 I : en unités arbitraires, F.I. = faisceau intégral, $X=$ dêpôt, $Y=$ zone de diffusion, $Z=$ acier.

Fig. 2 - Concentration profiles (G.D.O.S.). Deposits on 35 NCD 16 steel. $I=$ arbitrary units, F.I. = integral beam, $X=d e-$ posit, $Y=$ diffusion layer, $Z$ = steel. 
III.1 - Interactions avec WC $+30 \% \mathrm{Co}$.

Le bore diffuse dans le cobalt mêtallique de liaison et réagit avec lui. Les cristaux de WC sont progressivement dissous et des prêcipités apparaissent dans la nouvelle phase liante (Fig. 3 ). Ces processus déterminent une zone perturbée à la surface du substrat, dénommée "souscouche", dans laquelle le monoborure de cobalt (BCo) a été mis en évidence par diffractométrie des rayons $x$ pratiquée sur la surface des êchantillons. Des phases de type E (W-B-Co) décrites par ailleurs /4/, /5/ n'ont pas été détectées par cette technique, mais les examens à la microsonde confirment la présence de tungstène entre les grains de wC.

Cependant, à $1^{\prime}$ interface dépôt-sous-couche, se forme un film de 1 micron environ d'épaisseur (Fig.3), principalement constitué de BCo. Le tungstène n'apparait pas dans cette zone ainsi que dans le dépôt (microsonde).

on note par ailleurs une très faible migration du cobalt vers le carbure de bore. Le revêtement contient moins de $0,8 \%$ at. de cet élément très près de l'interface dépôt-sous-couche.

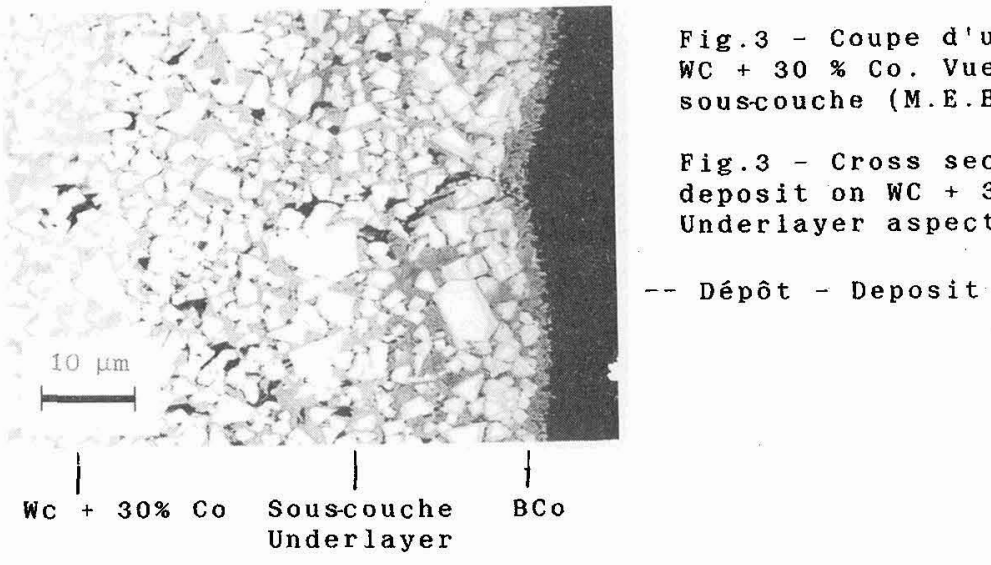

II -2 . Interactions avec 1 'acier 35 NCD 16 .

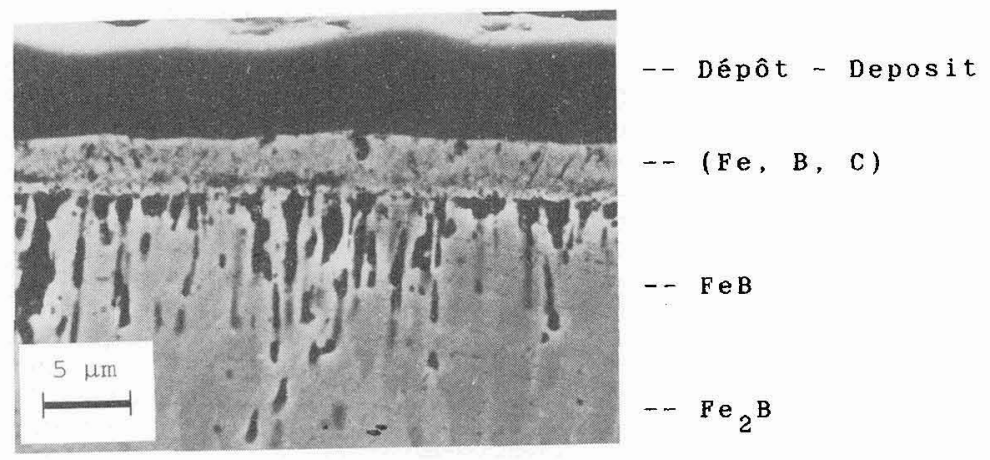

Fig.4 - Coupe d'un dépôt sur acier 35 NCD 16. Mise en évidence de la zone fragile (M.E.B.).

Fig. 4 - Cross section of a deposit on 35 NCD 16 steel showing the brittle layer (S.E.M.). 
Dans ce cas également, il se produit une diffusion rapide du bore dans l'acier avec formation des borures de fer Fe $B$ et FeB en couches successives comme dans le cas des borurations conventionnelles. Suivant les conditions de dépôt, on observe la présence d'une couche très poreuse et fragile (couche Fe, B, C) (Fig.4) constituée de fer, de bore et de carbone (Fig.2). L'analyse à la microsonde conduit à la formule globale suivante: Fe $B(0,9-0,95) \quad C(0,5-0,33)$, Ie nickel et le chrome étant à l'état de traces; le carbone semble provenir de la zone du substrat occupée par les borures de fer qui n'en contiennent pratiquement pas (Fig.2). La nature des phases présentes n'a pas pu être déterminée jusqu'à présent.

En conclusion, on observe dans tous les cas des diffusions importantes qui créent des zones de fragilisation nuisibles pour l'adhérence du revêtement.

on peut envisager de 1 imiter ces phénomènes par une barrière de diffusion disposée entre le substrat et le dépôt. Des essais réalisês avec le carbure de titane (TiC) ont donné satisfaction sur ce point ( Fig. 5 ).

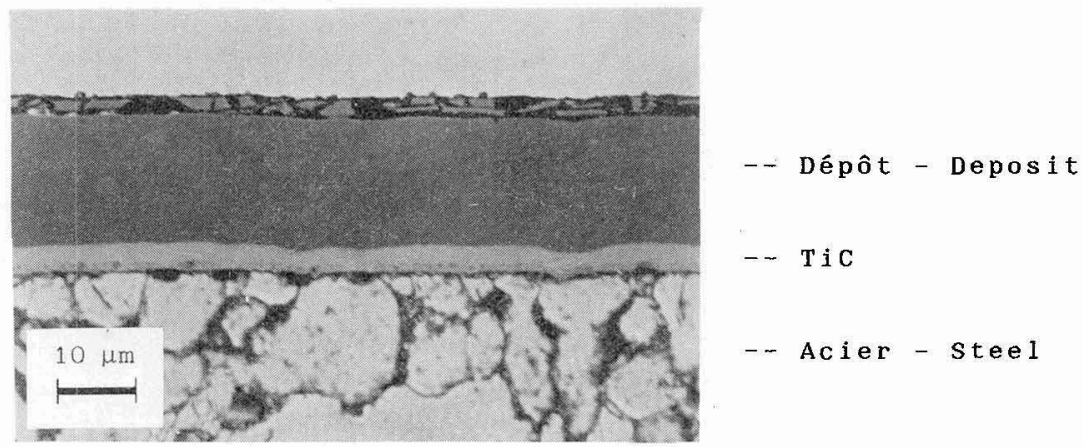

Fig.5 - Coupe d'un dépôt sur acier doux avec barrière de diffusion en TiC (M.O.)

Fig. 5 - Cross section of mild steel with a TiC diffusion barrier (O.M.).

IV - CARACTERISTIQUES DES COUPLES DEPOT-SUBSTRAT.

IV-1. Formation et adhérence des dépôts.

Sur les carbures de tungstène des revêtements homogènes et adhérents sont réalisés aisêment, dans les conditions opératoires du paragraphe II. Le comportement avec les aciers est différent car des conditions de dépôt bien définies sont nécessaires.

Dans le premier cas (WC + Co), la souscouche -par suite des fissurations qui apparaissent /3/- semble être le maillon le plus fragile du composite. L'écaillage quí en résulte, dans des conditions de contraintes sévères (forage de roche par exemple), est atténué lorsque la. teneur en liant du substrat est inférieure à $10 \%$. La figure 6 représente la coupe d'un dépôt réalisé sur $w C+6 \%$ Co dont la souscouche est peu perturbée et exempte de pores. 


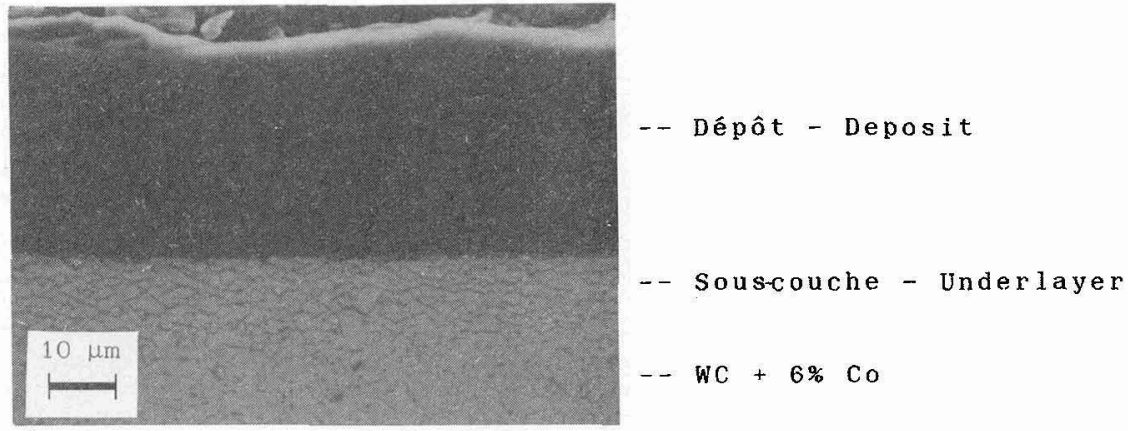

Fig.6 - Coupe d'un dépôt sur WC +6\% Co. (M.E.B.)

Fig.6 - Cross section of a deposit on WC +6\% Co (S.E.M.).

Sur acier, l'adhérence plutôt médiocre des dépôts est tributaire de l'existence de la couche fragile.(Fe, B, C) et de l'épaisseur du monoborure de fer qui provoque des fissurations au niveau des interfaces avec Fe B et le dépôt en raison de la grande différence des coefficients de dilatation thermique.

Une optimisation a cependant pu être faite par un plan d'expérience factoriel fractionnaire. Les résultats montrent qu'une très basse pression et un débit d'hydrogène élevé favorisent 1 a formation d'un dépôt adhérent. L'intérêt de la dilution du vecteur de bore par 1 'hydrogène pour minimiser la perte en fer du substrat due à la formation d'halogénures volatils avait été signalé par ailleurs/6/en relation avec la formation de zones poreuses.

La coupe d'un dépôt réalisé sur 35 NCD 16 dang les conditions optímales $\left(T=127 \underline{0}_{1} \mathrm{~K}, \mathrm{P}=2 \mathrm{mbar}, \mathrm{D}\left(\mathrm{BCl}_{3}\right)=11 \mathrm{~h}^{-1}, \mathrm{D}\left(\mathrm{CH}_{4}\right)=0,151 . \mathrm{h}^{-1}\right.$, $\left.\mathrm{D}\left(\mathrm{H}_{2}\right)=251 \cdot \mathrm{h}^{-1}, \mathbf{t}=1 \mathrm{~h}.\right)$ ne prêsente ni couche fragile (Fe, B,

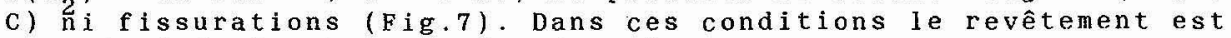
constitué de la phase quadratique $\mathrm{B}_{50} \mathrm{C}_{2}$.
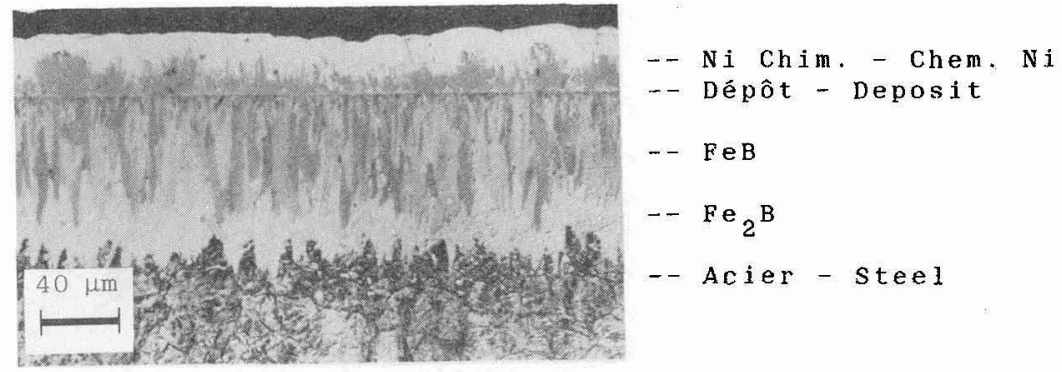

Fig.7 - Coupe d'un dépôt sur 35 NCD 16 réalisé à la suite d'un plan d'expériences (M.0.)

Fig.7 - Cross section of a deposit on 35 NCD 16 steel obtained from an experimental planing procedure (O.M.). 
IV-2. Microdureté et tests de forage.

Quelle que soit sa composition, le carbure de bore déposé est plus dur que les couches sous-jacentes comme le prouvent les exemples du tableau suivant :

\begin{tabular}{|l|c|c|}
\hline \multicolumn{2}{|c|}{ Microdureté Knoop sous $100 \mathrm{~g} \cdot$ de charge en $\mathrm{kg} \cdot \mathrm{mm}^{-2}$} \\
\hline & WC $+15 \%$ Co & Arier $35 \mathrm{NCD} 16\left(280 \mathrm{~kg} \cdot \mathrm{mm}^{-2}\right)$ \\
\cline { 2 - 4 } Substrat & 1270 & 650 \\
Souscouches & 1520 & 1500 \\
Dépôt & 3750 & 3600 \\
\hline
\end{tabular}

Des essais comparatifs sur banc de forage en milieu rocheux homogène ont été effectués. Les performances obtenues avec les carbures de bore sont nettement supérieures à celles des carbures seuls ou révêtus de TiC commercialement disponibles.

$\mathrm{V}-$ CONCEUSIONS.

De fortes interactions, qui conduisent à la formation de zones fragiles, ont Iieu lors du dépôt de carbure débore sur les carbures de tungstène ou les aciers, quelle que soit la nuance des substrats. La meilleure adhérence des dépôts de différentes compositions réalisés sur WC + Co est obtenue pour les plus faibles teneurs en cobalt. Sur acier, seuls des revêtements riches en bore $\left(\mathrm{B}_{50} \mathrm{C}_{2}\right)$ peuvent être réalisés de façon satisfaisante mais, même dans ce cas, l'utilisation d'une barrière de diffusion pour le bore semble constituer une meilleure solution.

Les déterminations de dureté et les tests de forage effectués sont encourageants et permettent d'envisager une exploitation future de ces revêtements /7/.

Les analyses à la microsonde électronique ont été effectués aux Laboratojres de I'U.E.R. de Nancy I, (54506, Vandoeuvre-les-Nancy, M. Claude), et la spectromêtrie à Décinarge Luminescente à I'I.R.S.I.D. (185; Avenue du Président Roosevelt, 78105, St.Germain en-Laye -R.Berneron).

\section{BIBI, I OGRAPHIE}

-/1/ Thevenot F., Bouchacourt M., Ind.ceram., 732, (1979), 655.

$-/ 2 /$ Beauvy M., Thevenot F., Ind.Ceram., 734, (1979), 811.

-/3/ Cartigue S., Cazajous D., Nadal M., Malé G., Proc.Conf.Euro cvD 8, June 17-20, 1985, Uppsala, Sweden.

-/4/ Bonetti R., Hintermann II.E., Proc. 6Th Int.Conf. on CVD,

Atlanta, The Electrochem.Soc.., Princeton, 1977, 351.

- /5/ Mercurio-Lavaud D., Petrov R., Peshev P., Zakhariev Z.,

J.Less. Common Met., 67, (1979), 59 .

-/6/Ramain S., Thesis, N¹13 B, Université de Perpignan, 1983.

-/7./ Ducarroir M., Malé G., Nadal M., Basse A.,Pizzini G., Cazajous D.,

"Procédé de réalisation de revêtements de carbure de bore et revê-

tements obtenus", Brevet $N^{\circ} 84.10290$, France, 27 Juin 1984. 